\title{
Design and Research of Virtual Payment System in Colleges and Universities
}

\author{
Sun Yang*, Lixia Wen \\ Jinan University, Guangzhou, China \\ Email: *shiqishidai1983@jnu.edu.cn
}

How to cite this paper: Yang, S., \& Wen, L. X. (2020). Design and Research of Virtual Payment System in Colleges and Universities. Open Journal of Social Sciences, 8, 455-464. https://doi.org/10.4236/jss.2020.86035

Received: May 27, 2020

Accepted: June 25, 2020

Published: June 28, 2020

Copyright $\odot 2020$ by author(s) and Scientific Research Publishing Inc. This work is licensed under the Creative Commons Attribution International License (CC BY 4.0).

http://creativecommons.org/licenses/by/4.0/

\section{(c) (i) Open Access}

\begin{abstract}
With the development of mobile payment and intelligent technologies, virtual payment system has been introduced to campus to improve the campus payment environment and the degree of intelligence on campus, so that online and offline campus payment can be combined, and payment by scanning QR code and card can be simultaneously achieved to greatly facilitate the learning and life of teachers and students, meet their diverse payment needs and enhance the service level and payment efficiency. Based on summarizing the problems faced by traditional campus card system in application, functions, and services, this paper analyzes the goals of building a virtual payment system, and builds a virtual payment system integrating applications, such as virtual campus card, electronic payment, and aggregation payment.
\end{abstract}

\section{Keywords}

Campus Card, Virtual Campus Card, Electronic Payment, Aggregation Payment

\section{Introduction}

With a large number of new demands of teachers and students springing up in school service management, and the rapid development of a new generation of information technologies such as "virtual card, aggregation payment, big data, artificial intelligence, blockchain and mobile Internet", the campus intelligence has been improving, bringing innovative changes to the functions and technology of the campus card system. For example, new applications including virtual card, electronic payment, and aggregation payment are constantly emerging, which enriches the campus payment life of both teachers and students.

Therefore, the traditional campus card system fails to meet the school's growing demand for mobile payment and mobile campus management. Under this 
background, it is necessary to build a virtual payment system that integrates the functional attributes of service fields such as the living, payment, learning, authentication of teachers and students to provide on-campus services represented by aggregation top-up, payment, canteen consumption, etc., public services represented by school payment and authentication, commercial services represented by consumption, and enhanced services represented by campus card big data, so as to optimize the functions and services of the campus card system in an all-round way.

\section{The Main Problems Faced by the Traditional Campus Card System}

The school's original campus card system still relies on physical cards, many campus card services depend on manual services, and have not yet been upgraded to mobile and virtual payment. Meanwhile, a unified payment platform has not yet been established for on-campus payment. At present, the infrastructure and the expansion capability can no longer meet the needs for functional expansion in the new era, and fail to effectively support the top-level design of informatization. Besides, the long service life of hardware tools will inevitably result in potential stability problems.

\section{Goals of Virtual Payment System Construction}

By building a "convenient, efficient and high-quality" virtual payment system, the campus payment services can be standardized, the efficiency of financial and management personnel can be comprehensively improved, and convenient campus card services can be provided through a unified payment application, mobile services and multi-media and multi-channel payment methods, which allow more traditional applications to be solved automatically through the system, to better serve the users and administrators of campus cards, and to solve the consumption applications of smart campus informatization.

The construction of online payment platform based on the school's existing campus card payment and management system will greatly improve the system's ability to process highly concurrent services. The school's original campus card accounts are integrated with electronic accounts to build unified electronic accounts in the school. The expanded virtual payment platform will serve as a unified on-campus payment platform to manage the consumption on campus in a uniform manner, and provide various payment methods such as physical cards, virtual cards, WeChat, Alipay, UnionPay, and bank payment. Users can realize offline consumption in canteens, supermarkets and other places, and have access to online payment services such as online top-up, payment, and reservation through the accessed payment methods.

\section{Design of the Virtual Payment System}

\subsection{Overall Structure of the Virtual Payment System}

Based on the campus card payment and management system, and the Internet 
financial standards, the system adopts distributed architecture design (Figure 1). 1) Flexible technological architecture of payment channels can support connection to interfaces of various mainstream payment technologies, including the PC browser payment, mobile browser payment, QR code payment, subscription payment, mini program payment, and password-free payment of major banks and various payment institutions. 2) Powerful payment project management system supports multi-level structure management of departments, supports multiple payment projects of one department, controls available payment channels (payment technologies) according to payment project, and supports payment institutions to directly clear payment projects. 3) Automatic channel reconciliation mechanism supports automatic reconciliation with payment channels, which facilitates the discovery and handling of unbalanced accounts. 4) Perfect fund settlement system for payment projects supports automatic or manual settlement. Automatic settlement can be conducted on a daily, weekly, monthly basis, and supports settlement review. It also supports manual fund carried forward, and can connect to bank transfer interface to achieve automatic carried forward to bank account, or connect to the school's financial system interface to realize automatic carried forward to the financial projects. 5) Complete payment records and support of transaction refund. Payment records can be inquired according to multiple dimensions such as collection unit, payment items, payment methods, payment types, settlement status, date and time, and the payment records can be summarized and analyzed. 6) With perfect account management system, accounting documents can be generated for all transactions in accordance with the Government Accounting Systems, and current accounts can

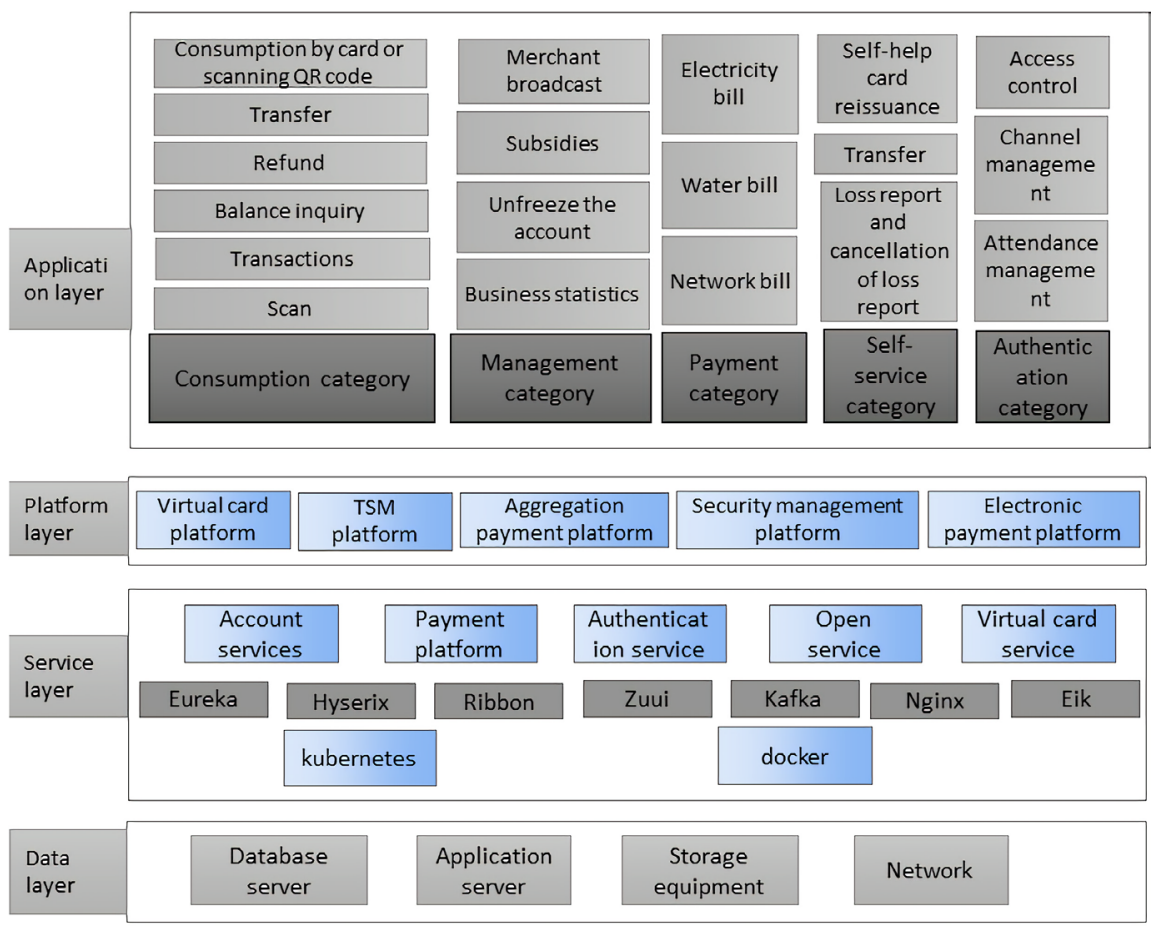

Figure 1. Overall architecture of the virtual payment system. 
be used to track the funds of receivable channels and payable projects. 7) (Yuan, 2020) The system provides a secure open platform which supports third-party systems to connect payment interface to access electronic payment platform, and supports authentication of third-party applications, permission authentication of third-party payment projects, and multi-terminal secure payment agents. In addition, the open platform provides interfaces such as PC cashier, mobile cashier (H5), authorization code payment, mini program payment, download of statement of account, transaction inquiry, transaction cancellation, transaction closure, refund application, and refund inquiry.

The overall campus card platform is divided into three layers: the infrastructure layer, the core framework layer, and the service platform layer. The three layers are designed based on the concept of "high cohesion and loose coupling".

1) Infrastructure layer: it includes physical hardware equipment such as the upgrading of the overall architecture of the data center, campus card VLAN network and servers, and virtualized system software.

2) Core framework layer: it is a complete set of micro-service architecture based on VM, Kubernetes and Docker container technology, and Spring-Cloud framework. It provides strong guarantee for the concurrency, fault-tolerance, and throughput of open service platform, APP mobile services, and virtual campus card platform.

3) Service platform layer: it includes the services platforms such as aggregation payment, virtual card, electronic payment, identity authentication, campus card application, etc. Each service platform is relatively independent but also cooperates with each other to provide professional support for front-end application systems, which realizes resource sharing and collaboration on services.

The aggregation payment platform which adapts to the development of the technology of campus card application system, supports the native QR codes of Alipay, WeChat, banks, and UnionPay, and multiple medias such as physical cards and QR codes. Based on this upgraded platform, other medium such as face, finger veins, and iris can be expanded.

\subsection{Virtual Campus Card, Achieving Using Campus Card by Mobile Phone}

By building virtual campus cards based on WeChat, users can enter the campus card page through WeChat Cards \& Offers, Mini Programs, or Subscriptions. The virtual campus cards can be regarded as an online expansion of the offline physical campus cards, thus improving the use scenarios of campus cards will bring users great convenience. The WeChat payment code can be linked to the payment code application system of the virtual card management platform to display a dynamic QR code with payment function. A validity period can be set for each QR code (generally 45 seconds), and the QR code will automatically refresh after expiration, ensuring that the QR code will not used through screenshot. The campus card accounts and electronic accounts are combined into the electronic accounts with a complete account management system, which elimi- 
nates the reconciliation problems caused by the unbalance between card balance and library balance of the system, thereby greatly reducing the financial workload and error rate, and enhancing the efficiency of the administrators.

The virtual campus card establishes the main scenario of canteen where fast small-amount payment can be realized under the condition of offline with the payment link, ensuring the ability to cope with the weak network environment. Building a comprehensive payment mode with online payment as the main method and offline payment through POS as a supplement not only better serves the teachers and students of the school, but also regulates financial management and eliminates the management cost of cash charges. The construction of the application scenarios of campus code with identity recognition feature covers access control via code scanning, and conference sign-in, etc.

\subsection{Electronic Payment Platform, Realizing Online Consumption}

Establishing a unified payment platform and providing a standard payment interface provide online transaction services for third-party systems or applications through the web and $\mathrm{H} 5$, which solves the problems of traditional payment such as inconvenience in calling and weak scalability, reducing the difficulty of connection by third-party platforms. The clearing and processing modes of online payment accounts remain unchanged, and support linkage with the financial system to achieve automatic merchant clearing.

Features of the electronic payment platform: 1) Third-party access: multiple payment modes with flexible selection; unified interface standard facilitates connection; perfect authorization management improves security. 2) H5 scheme design: supports APP/WEB/WeChat/BS multi-terminal services simultaneously to allow the services to easily expand, realize the sharing of each terminal service, appeal to users' habits, and improve user experience. 3) For teachers and students: it supports online payment to meet the service needs of a mobile campus; supports multiple payment methods to meet users' different payment needs and enhance user experience. 4) For merchants: flexible self-service settlement method with automatic clearing and settlement functions; online payment reduces the purchase of POS equipment and saves costs; it shows the real transaction situation, and orders can be clearly inquired.

\subsection{Aggregation Payment Platform, Achieving Efficient Payment Mode}

It solves the fragmentation of online and offline payment scenarios on campus (multiple QR code scenarios), realizes automatic reconciliation, provides one-stop payment services based on the virtual campus card solution to support integration with third-party payment platforms such as WeChat, Alipay, UnionPay, and banks. The aggregation payment service is featured by multi-code integration, automatic reconciliation, multi-institution aggregation, adaption to user's habits, card-free payment, and full online and offline coverage (Yang, 2019).

A complete aggregation payment platform consists of functional modules 
such as payment gateway, callback gateway, timed task, and management background etc. (Figure 2) 1) Payment gateway is responsible for all interactions with banks or third-party payment platforms, including payment, signature verification, refund, query, and bill download. 2) Callback gateway is responsible for receiving the asynchronous payment result and refund result notifications of banks or third-parties payments. 3) Timed task is mainly to take the initiative to obtain payment and refund results, because the asynchronous callback notifications of banks or third-party payments are sometimes delayed for a long time, the payment results cannot completely rely on the third-party payment notifications. The platform actively inquires the payment result of the order on the third-party platform every second. 4) Management background supports payment result query, refund result query, and routing rule management, etc.

Flow chart of POS consumption through native QR code of WeChat or Alipay.

\subsection{Interface Authorization, Providing Open Interface}

The interface authorization (TSM) is mainly served to provide standardized and unified interface standards and authorization management mechanism for third-party payment companies, application systems, and hardware equipment that use campus payment services to ensure transaction security and provide open transaction use and access services (Li, 2019). Providing open API interface to share campus card service helps the school to establish a more reasonable management and service mechanism. The interface authorization management module introduces the management of API, ensuring the safe deployment, distribution, management and monitoring of API, so that the entire API ecosystem

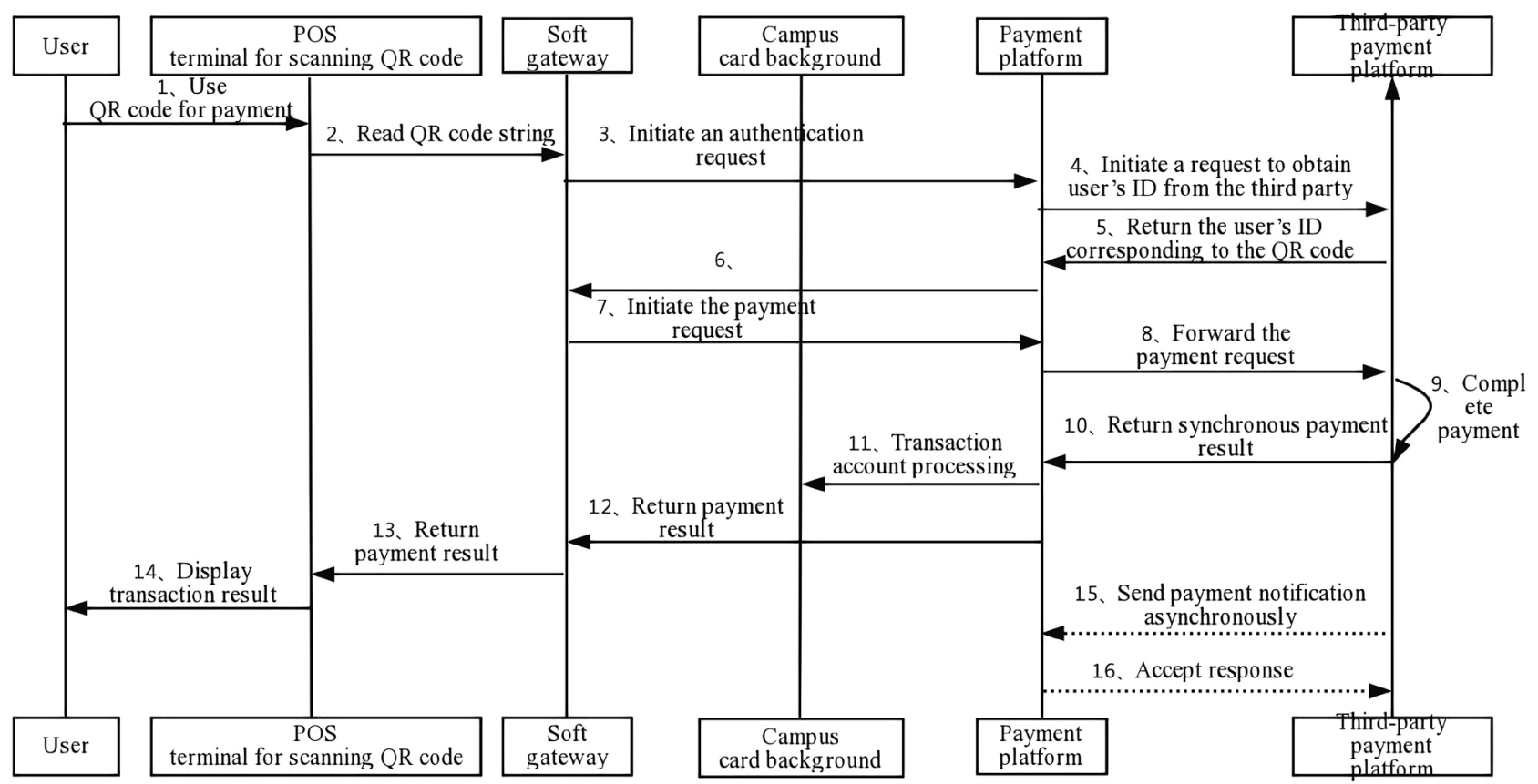

Figure 2. Identity authentication and consumption transaction in aggregation payment. 
can develop in a more healthy and orderly manner. The TSM platform downloads documents from WeChat, Alipay, banks, and UnionPay via SFTP to perform local reconciliation, and generate reconciliation reports.

The TSM platform provides the following standard interfaces:

1) Basic interfaces of campus card: query, loss report, cancellation of loss report, top-up, etc.

2) Transfer interface of campus card: the interface for top-up direct card writing.

3) Unified ID authentication interface: related interface for ID authentication.

4) Payment interfaces: electronic account payment interface, WeChat payment interface, Alipay payment interface, and UnionPay payment interface, etc.

5) Payment interfaces: payments of electricity bill, network bill, water bill, tuition fee, etc.

6) Access Control \& Attendance interfaces: interfaces for access control and attendance query.

7) Interfaces of orientation registration and graduation system: related interfaces for orientation registration and graduation system.

8) Third-party application system interfaces: corresponding interfaces will be developed subsequently according to the actual application system.

\subsection{Fund Settlement, Realizing Convenient Financial Management}

There are two main transaction modes of consumption transactions in the virtual payment system, namely, campus card electronic account transaction and aggregation payment transaction. The transaction reconciliation and merchant settlement of the two transaction modes are completed by the same platform of the virtual payment system, which reduces the reconciliation and settlement workload of financial personnel.

The electronic account transaction mode requires users to top up the electronic account firstly by cash, bank transfer, WeChat, and Alipay; when users use physical cards and virtual cards for consumption on campus, funds will be deducted from the electronic account balance (Liu, 2020). The virtual payment system automatically completes the reconciliation with top-up channels. Under the aggregation payment transaction mode, users can use native QR code of WeChat and Alipay to consume on POS, and the virtual payment system decodes to initiate transaction request to WeChat and Alipay. The funds are automatically transferred to the school's account after the transaction is completed, and the virtual payment system automatically reconciles with third-party payment channels such as WeChat and Alipay. Both modes automatically generate settlement reports based on the user's consumption, and support automatic settlement and self-service settlement of merchants. From the perspective of absolute security, the self-service settlement mode is usually used. The financial department regularly settles funds to merchants according to the settlement reports generated by the system. 


\subsection{Other Applications}

Aggregation code system: it realizes merchant's aggregation code. Merchants can print aggregation code, so that users can pay by the native scanning of WeChat, Alipay, and UnionPay. The aggregation code is actually a QR code that contains multiple collection links. When the user scans the QR code, the background will automatically recognize the payment interface used by the user.

Merchant consumption terminal: replaces terminal equipment that supports payment by both card and code, replaces desktop LCD cash registers, hanging LCD cash registers, switches, etc.

Terminal management: the terminal management module is the soft gateway, which provides transaction access and processing mechanism for all terminal equipment used (such as consumption POS), and performs authorization management on the equipment. The soft gateway is responsible for the list management, current account recovery, and parameter download of cash register.

\section{Application Scenarios of the Virtual Payment System}

The school's existing WeChat platform is upgraded to the WeChat campus platform, which integrates a series of services such as virtual card services, campus card services, payment services, query services, venue reservation, online meal ordering using $\mathrm{H} 5$ technical solution and the combination of application modules, to provide campus card services and peripheral extension services including online query, online payment, online reservation, and account management, so that users can be provided with a unified service entrance to access more convenient, fast and efficient one-stop integration services.

Payment services: payments for electricity bill, network bill, minor courses, grade examinations, Party membership dues and League membership dues, training, tuition, etc. Among them, electricity, water and network bills are connected to the school's water and electricity control system and network payment system to realize payment services; minor courses fees, grade examinations fees, Party membership dues and League membership dues, training fees and tuition fees can be paid by accessing the schools' existing collection system.

Reservation services: by adopting $\mathrm{H} 5$ technical solution, meal reservation, venue reservation, school bus reservation and other services can be integrated.

Merchant services: application services in different scenarios can be provided according to the actual needs of merchants. In order to allow merchants to keep track of the transactions at any time, they are provided with a mobile-end transaction inquiry application, so that they can perform transaction inquiries according to POS terminals and dates. In order to enable merchants to avoid frequent checking of the transaction results when the transactions are made through scanning, merchants are provided with a broadcast application, so that the transaction results can be broadcast in real time. The broadcast types include the payments made by scanning merchant's collection QR code, or by scanning consumer's payment QR code, or both. Optional terminal broadcast can be available for the merchants with multiple terminals (multiple POS). 


\section{Advantages and Limitations of Virtual Payment System}

The advantages of the virtual payment system are as follows:

From the perspective of users: unified account and only one payment wallet help to understand clearly; it supports multiple payment methods to meet different payment needs of users conveniently and quickly. Student and teachers can choose the payment method they are used to via the mobile phone/PC terminal to consume and pay in the school; the user experience can be improved.

From the perspective of management: multiple payment methods can be provided at the same time by one-time access, and the integration of card accounts and electronic accounts simplifies account management. Unified platform for payment and reconciliation contributes to simple and efficient financial management.

From the perspective of merchants: it supports consumption by scanning QR code, and provides more payment methods; the trading information can be inquired at any time to help the merchants keep track of the income in real time.

Others: multiple payment modes, flexible application; unified interface reduces difficulty of connection; perfect authorization management ensures security.

However, the virtual payment system still has limitations, and the face recognition payment method should be considered in the future. With the progress in biometric recognition technology, face recognition payment will become a new payment trend. The mobile payment process requires mobile phone, and many consumers will encounter the situation where the mobile phone is out of power or they forget to carry the mobile phone, while the face recognition payment does not require a mobile phone since the payment can be completed only by face recognition.

\section{Conclusion}

The construction of the virtual payment system will improve the campus payment and campus card system to a new level, and effectively enhance the school's information service level and brand influence; diverse payment methods and medias provided by the school can effectively stimulate the consumption enthusiasm of teachers and students in the school, and build a standardized payment platform to improve the effectiveness of financial management at the same time. In terms of teaching, it can provide data support for the school to analyze students' daily behaviors, enabling the school to accurately assess poor students with sufficient consumption data, and can provide multiple payment channels for teachers and students to pay for various bills; self-service automatic financial reconciliation, clearing, and settlement can reduce the work of financial personnel; online services such as card handling, top-up, loss report, cancellation of loss report, and payment, etc. reduce the workload of staff in card center, and improve the service experience of teachers and students. Regarding students' life, students can use the original campus cards, virtual cards, and native QR codes of WeChat, Alipay, UnionPay, and banks to pay in canteens, super- 
markets, shops, school hospitals, swimming pools and other consumption places; in ID recognition scenarios, such as libraries, dormitories, school gates, they can use the original campus cards, virtual cards and other medias, without worrying about forgetting taking the card.

The virtual payment system has a complete account management system, and provides online and offline payment services simultaneously. Cardholders can enjoy campus payment services through cards, QR codes and other methods, which truly breaks the media restrictions of campus cards. School service departments, merchants, and third-party applications can easily and quickly access and use payment services. Unified management will be implemented for accessed banks and third-party payment platforms, and the campus payment services can be extended to desktop, mobile and self-service terminals, thereby building a campus payment ecosystem.

\section{Fund}

This paper is the phased result of Guangzhou Science and Technology Innovation and Development Special Fund Project (2019) "artificial intelligence-based human-computer interaction intelligent learning platform development and application", Project Grant No.: 201902010041.

\section{Sponsored}

The paper reported here is sponsored by 2019 Guangdong University's Party Construction Research Project, Project name: Research on the Innovation of Party Construction in Colleges and Universities in the New Era, Project Grant No.: 2019BK072.

\section{Conflicts of Interest}

The authors declare no conflicts of interest regarding the publication of this paper.

\section{References}

Li, C. B. (2019). Design and Implementation of Virtual Campus Card System. Computer Programming Skills \& Maintenance, 1, 35-37.

Liu, X., \& Wu, J. (2020). Design of Campus Card APP Based on Campus Card. China New Telecommunications, 2, 107.

Yang, T., Zhao, L., \& Li, X. (2019). Financial Technology and Payment Reform (Technology, Mode and Account).

Yuan, Y. Y., Wang, Q., \& Yan, C. X. (2020). Investigation and Analysis of Virtual Campus Card Acceptance Behavior Based on TAM Model Structure. China Collective Economy, 2, 79-81. 